\title{
Imaging and Therapeutic Applications of Optical and Thermal Response of SPION-Based Third Generation Plasmonic Nanodendrimers
}

\author{
Maryam Tajabadi', Mohammad E. Khosroshahi ${ }^{1,2 *}$, Shahin Bonakdar ${ }^{3}$ \\ ${ }^{1}$ Laser and Nanobiophotonics Laboratory, Biomaterial Group, Faculty of Biomedical Engineering, Amirkabir \\ University of Technology, Tehran, Iran \\ ${ }^{2}$ Center for Advanced Diffusion-Wave Technologies (CADIFT), Department of Mechanical and Industrial \\ Engineering, University of Toronto, Toronto, Canada \\ ${ }^{3}$ Pasteur Institute of Iran, National Cell Bank of Iran, Tehran, Iran \\ Email: ${ }^{*}$ hhosrom@mie.utoronto.ca
}

Received 4 June 2015; accepted 24 July 2015; published 27 July 2015

Copyright (C) 2015 by authors and Scientific Research Publishing Inc.

This work is licensed under the Creative Commons Attribution International License (CC BY).

http://creativecommons.org/licenses/by/4.0/

c) (i) Open Access

\section{Abstract}

In this study, $9 \mathrm{~nm}$ superparamagnetic iron oxide nanoparticles (SPION) were functionalized by polyamidoamine (PAMAM) dendrimer. Using tetracholoroauric acid $\left(\mathrm{HAuCl}_{4}\right)$, magnetodendrimer (MD) samples were conjugated by gold nanoparticles (Au-NPs). Two different reducing agents, i.e. sodium borohydride and hydrazine sulfate, and pre-synthesized 10-nm Au-NP were used to evaluate the efficiency of conjugation method. The samples were characterized using X-ray diffractometry (XRD), transmission electron microscopy (TEM), Fourier transform infrared (FTIR) spectroscopy, UV-visible spectroscopy and fluorescence spectroscopy. The results confirmed that AuNPs produced by sodium borohydrate and the pre-synthesized 10-nm Au-NPs were capped by MDs whereas the Au-NP prepared by hydrazine sulfate as a reducing agent was entrapped by MDs. Optical properties of the MDs were studied by laser-induced fluorescence spectroscopy (LIF) within a wide range of visible spectrum. Also, based on the thermal analysis, all synthesized nanostructures exhibited a temperature increase using $488 \mathrm{~nm}$ and $514 \mathrm{~nm}$ wavelengths of a tunable argon laser. The new iron oxide-dendrimer-Au NPs synthesized by sodium borohydrate (IDA$\mathrm{NaBH}_{4}$ ) produced the highest temperature increase at $488 \mathrm{~nm}$ whereas the other nanostructures particularly pure Au-NPs produced more heating effect at $514 \mathrm{~nm}$. These findings suggest the potential application of these nanocomposites in the field of bioimaging, targeted drug delivery and controlled hyperthermia.

\footnotetext{
${ }^{*}$ Corresponding author.

How to cite this paper: Tajabadi, M., Khosroshahi, M.E. and Bonakdar, S. (2015) Imaging and Therapeutic Applications of Optical and Thermal Response of SPION-Based Third Generation Plasmonic Nanodendrimers. Optics and Photonics Journal, 5, 212-226. http://dx.doi.org/10.4236/opj.2015.57021
} 


\section{Keywords}

\section{SPIONs, PAMAM Dendrimer, Gold Nanoparticles, Fluorescence, Bioimaging, Therapy}

\section{Introduction}

Recently a variety nanomaterials including magnetite superparamagnetic iron oxide nanoparticles (SPION) and nobel metal nanoparticles such as gold have been the focus of many research fields particularly in medicine and biomedical engineering which has shown a promising advancement [1]-[3]. Plasmonic nanoparticles exhibit unique optical properties with major advantages due to the photophysical properties: strong localized surface plasmon resonance (LSPR), surface-enhanced scattering, non-linear optical properties, tunable resonance across the Vis-NIR due to adjustable nanoparticle size and shape, biocompatibility due to their inert surface, nontoxicity, surface conjugation chemistry, i.e., they can be linked to specific ligands for tumor targeting, imaging and therapies, lack of photobleaching or blinking as with quantum dots, and very low oxidation [4]-[9]. Not only can gold nanoparticles (Au-NPs) operate as an optical signal transfer in plasmonic devices but also they can be used as a useful platform for analytic-receptor interaction [7] [10]. Au-NPs need surface treatment with different molecules to precisely perform desirable applications. There are some evidence of Au-NPs modification with silica, liposome and linear polymers [3] [9] [11] [12].

Researchers explicitly assert that poly(amidoamine) (PAMAM) dendrimers can act as a template or stabilizer for preparation of inorganic nanocomposites. The resultant nanocomposites have much applicable potential such as gen vector, catalysis, resonance imaging agents and nanocapsules [6] [7] [13] [14]. Since dendrimers have unique chemical structures, molecular weight and molecular size which can provide special type of functionality, they receive privileged attention in developing fields of materials science [15]-[18]. Considering large number of terminal groups on the exterior of the molecule and interior voids, dendrimers introduce as an attractive platform for metal ion celates [19]. Indeed, dendrimer-entraped inorganic nanoparticles (DENP) include a nanostructure where one or more inorganic nanoparticles with the diameter of less than $5 \mathrm{~nm}$ are entrapped within an individual dendrimer molecule. In the case of dendrimer-stabilized inorganic nanoparticles (DSNP), one inorganic nanoparticle which usually has the diameter larger than $5 \mathrm{~nm}$ is stabilized by multiple dendrimer molecules [20]. Evaluation of formation of both DENP and DSNP in the presence of amine-terminated PAMAM dendrimers with and without addition of reducing agents has been the subject of several studies over the past few years [13] [21]-[23]. Most of chemical methods of Au-NP preparation include nucleation and then growth of gold clusters by reduction of a gold salt (20). The bond is formed between gold nanoparticles and dendrimers as a result of interaction between coordinating groups such as $-\mathrm{OH},-\mathrm{NH}_{2}$, or $-\mathrm{COOH}$ and gold ions [24].

The contemporary issues in cancer treatment are production of effective nanoprobes for tumor targeting and selective therapy [5] [25]. A fine magnetite nanoparticles with simple production method, nearly uniform size distribution and high magnetization saturation were prepared in our previous study [3] [12] [25]-[27], the optimal magnetite nanoparticles were chosen, coated with third generation PAMAM dendrimer and finally functionalized with Au-NP via different preparation methods. The main intention of the paper was to synthesize third generation dendrimers conjugated with Au-NPs using three methods of sodium borohydrate, hydrazine sulfate as the reducing agents and pre-synthesized Au-NP, and compare their fluorescent and optothermal properties with dendrimers for the purpose of biomedical application.

\section{Materials and Methods}

\subsection{Materials}

Iron chlorides (III) $\left(\mathrm{FeCl}_{3} \cdot 6 \mathrm{H}_{2} \mathrm{O}\right)$, Iron Sulfate $\left(\mathrm{FeSO}_{4} \cdot 7 \mathrm{H}_{2} \mathrm{O}\right)$, and Ethanol $\left(\mathrm{C}_{2} \mathrm{H}_{5} \mathrm{OH}\right)$ were purchased from Merck Company. Ammonium Hydroxide $\left(\mathrm{NH}_{4} \mathrm{OH}\right)$, Methyl Acrylate $\left(\mathrm{CH}_{2}=\mathrm{C}\left(\mathrm{CH}_{3}\right) \mathrm{COOCH}_{3}\right)$, Ethylenediamine $\left(\mathrm{C}_{2} \mathrm{H}_{4}\left(\mathrm{NH}_{2}\right)_{2}\right)$, Soduiom Borohydrate $\left(\mathrm{NaBH}_{4}\right)$, Hydrazine sulfate $\left(\mathrm{H}_{6} \mathrm{~N}_{2} \mathrm{O}_{4} \mathrm{~S}\right)$, Gold colloid solution (10 $\mathrm{nm})$, and tetracholoroauric acid $\left(\mathrm{HAuCl}_{4}\right)$ were obtained from Sigma Aldrich. 


\subsection{Formation of Gold and Magnetodendrimer}

Dendrimer grafted magentite nanoparticles (magnetodendrimers-MDs) were synthesized based on our previous published report [28]. The gold coated MDs were synthesized using the three aforementioned procedures to achieve the following objectives: i) to have efficient method of loading Au-NP, ii) to improve the fluorescence properties of material, and iii) to find the optimal optothermal properties.

a-Using sodium borohydrate (IDA- $\left.\mathrm{NaBH}_{4}\right)$ : A solution of tetracholoroauric acid $\left(\mathrm{HAuCl}_{4}\right)(5 \mathrm{mM})$ was prepared and added to the suspension of third generation magnetodendrimer $(1 \% \mathrm{w} / \mathrm{v})$ with the same volume under $\mathrm{N}_{2}$ atmosphere. In order to produce the complex between $\mathrm{Au}$ (III) and amide or amine group of dendrimer, the resulting mixture was vigorously stirred for one hour at darkness. After that, $5 \mathrm{~mL}$ of aqueous sodium borohydrate solution $(0.1 \mathrm{M})$ was added drop wise to the reaction mixture. The following reactions lead to reducing $\mathrm{Au}$ (III) to zero charge $\mathrm{Au}(0)$ nanoparticles. The reaction was continued under a vigorous stirring for 2 hours at $25^{\circ} \mathrm{C}$. The obtained nanoparticles are referred as IDA- $\mathrm{NaBH}_{4}$. These particles were rinsed with ethanol five times using magnetic separation. The same procedure was repeated with the tenfold concentration of $\mathrm{HAuCl}_{4}$ and to evaluate the completion of the reactions, a UV-vis analysis was performed at $0,15,30,45,60$, 90, and 120 minutes after addition of reducing agent. The product is referred as (IDA-10).

$\mathrm{b}$-Using hydrazine sulfate (IDA-Hydr.): The stable gold nanoparticles were produced by adding hydrazine sulfate $\left(\mathrm{H}_{6} \mathrm{~N}_{2} \mathrm{O}_{4} \mathrm{~S}\right)$ aqueous solution $(25 \mathrm{mM})$ to the solution of magnetodendrime- $\mathrm{HAuCl}_{4}$ complex and stirring for 2 hours. The particles were rinsed with ethanol five times as before and the sample was named IDA-Hydr.

c-Using gold nanoparticles (IDA-NP): Au-NPs (purchased from sigma Aldrich) were directly added to magnetodenrimer suspension under $\mathrm{N}_{2}$ atmosphere. The mixture was stirred for an hour to complete the reaction and then particles rinsed five times with ethanol.

\subsection{Characterization}

Crystalline phase of nanoparticles was confirmed using X-ray diffraction with radiation of $\mathrm{Cu} \mathrm{K} \alpha$ (XRD, $\lambda=$ $0.15406 \mathrm{~nm}, \mathrm{FK} 60-40$ X-ray diffractometer). The presence of PAMAM, gold formation on the surface of magnetite nanoparticles was proved by Fourier transform infrared (FTIR) spectroscopy (BOMEM, Canada). Particle size and morphology of nanocomposites were determined by transmission electron microscopy (TEM, Philips CM-200-FEG microscope, $120 \mathrm{kV}$ ). The amount of Au-NP attached to the magnetodendrimer was estimated using wavelength-dispersive X-ray spectroscopy (SEM-WDX, XL30, Philips, USA). The UV-vis spectra of nanoparticles were recorded using spectrophotometer (UV-2600, Shimadzu, Japan).

\subsection{Experimental Setup}

The evaluation of fluorescence emission of ID (G3), IDA-10, IDA-Hydr, and IDA-NP nanoparticles at different excitation wavelengths was performed using an ion argon laser Melles Griot-35MAP431) at $25^{\circ} \mathrm{C}$. The fluorescence signals were detected by a $600 \mu \mathrm{m}$ core diameter optical fiber (LIBS-600-6-SR, Ocean Optics) connected to spectrometer (UV-Vis USB 4000, Ocean Optics), see Figure 1. In our case, the excitation wavelengths were 454, 457, 465, 472, 477, 488, 496, 502 and $514 \mathrm{~nm}$. After obtaining the spectra of samples, they were smoothed by Gaussian model using Findgraph software.

The next set up was to evaluate the efficiency of these nanoparticles in coloring the polymeric substrate, using two kinds of natural polymers, i.e., cotton and collagen. After the injection of nanoparticle solution to these substrates, fluorescence microscopy (Zeiss Axioshop-Germany) was used to study the materials colouring.

\section{Results and Discussion}

\subsection{UV-Vis Evaluation}

The concept of dendrimer nanocomposites is based on immobilization of pre-organized metallic ions [29]. With respect to this concept, a dendrimer acts as a template or reactor to pre-organization of ions and small molecules [30]. The full generation of dendrimeric nanoparticles is electron donor in aqueous medium and form a cation. In such cases, atoms and molecules could attach to internal space or external surface of dendrimer [10] [31]. This pre-organization can lead to attachment of precursor-dendrimer, which is a dynamic equilibrium between template and reactants. The dynamic equilibrium causes a homogeneous distribution of ions and molecules. At 


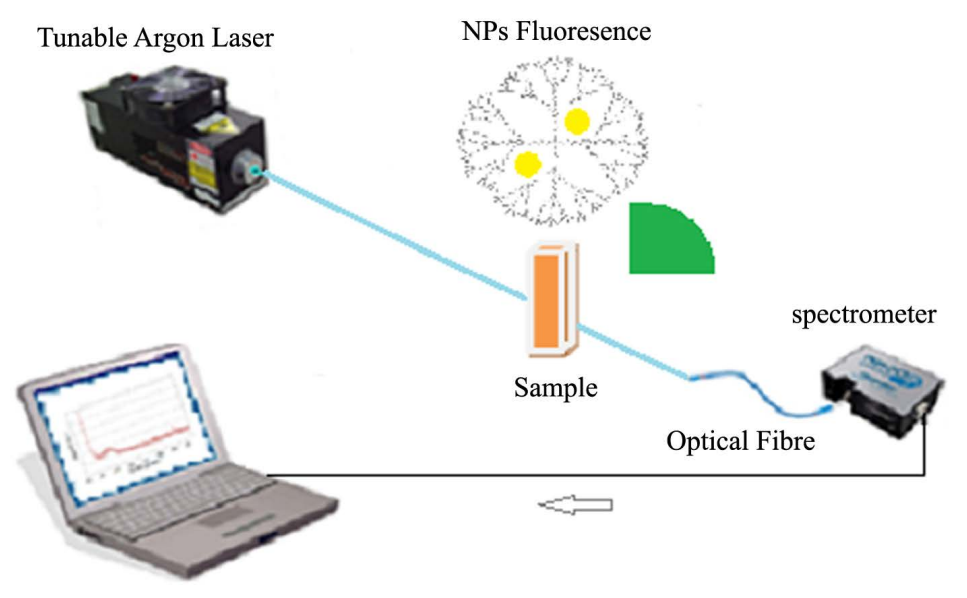

Figure 1. Experimental setup of laser-induced fluorescence spectroscopy for IDA nanocomposites.

the second stage, a series of reactions lead to production of resultant hybrid materials. At this stage, mostly a reducing agent is added to precursor-dendrimer complex and the complex loses $\mathrm{HCl}$ moieties and Au-NPs are stabilized in the dendrimer structure [30] [32] [33]. There are three different types of hybrid materials; i.e. internal, external and mixed type.

In this work, in order to evaluate the formation of Au-NPs during the synthesis reaction, sampling was done at different reaction times and the UV-vis spectra of these samples were recorded. Figure 2 indicates that all samples have a unique absorption peak in the range of 520 - $540 \mathrm{~nm}$ [10] [34], which reveals the formation of AuNPs. Plasmonic peak around $520 \mathrm{~nm}$ corresponds to the collective oscillation of gold free electrons [35]. During the synthesis reaction, the intensity of plasmon resonance peak was increased over time and the peak shifted toward higher wavelengths with respect to increase in particle size.

At synthesis condition of dendrimer-gold nanocomposite and in the presence of $\mathrm{HAuCl}_{4}$ molecules, amine group loses electrons and electrostatically interacts with $\mathrm{AuCl}_{4}{ }^{-}$ions. In $\mathrm{UV}$-vis spectrum of the condition prior to addition of reducing agent, an absorption peak was observed around $280 \mathrm{~nm}$ which is the characteristic peak of bound formation between $\mathrm{AuCl}_{4}^{-}$and dendrimer [36]. After addition of reducing agent, a broad peak around $520 \mathrm{~nm}$ was seen. This plasmonic peak is related to the collective oscillation of electrons [35]. Other researches indicate that peaks at 280 and $320 \mathrm{~nm}$ represent the aggregation of Au-NPs [10] [37] [38]. It should be mentioned that location and shape of surface plasmon resonance is strongly dependent on the particle size of material [39] [40] and the particle aggregation results in reduction of gold plasmon band intensity [38]. As it is seen in Figure 3, the peak ratio of $520 \mathrm{~nm}$ to $280 \mathrm{~nm}$ increases over the time which indicates the formation of Au-NPs. However, this ratio shows a reducing trend after 90 minutes which can be an indication of probability of aggregation and cluster formation of Au-NPs. No significant peak corresponding to Au NPs was observed in the UV-vis spectra shown in Figure 4 after purification of resultant material which may suggest that the final structure contains discrete gold nanoparticles (i.e. trapped) in a dendrimer substrate. This phenomenon was previously reported by [36] [41] [42].

\subsection{TEM Analysis}

Figure 5 indicates that the nanocomposites synthesized using $\mathrm{NaBH}_{4}$ are larger than those synthesized using hydrazine sulfate. Based on these results and other researcher suggestions, on the presence of $\mathrm{NaBH}_{4}$, the ions interact with surface amine group of magnetodendrimer [23] [38] [40] [43] and forms larger particles in the expense of smaller particles [35] [40]. When hydrazine sulfate was used as a reducing agent, the homogeneous distribution and smaller particles were obtained which could be explained as an entrapment and formation of Au-NPs on the cavity of magnetodendrimer [43].

\subsection{XRD Analysis}

In order to evaluate the presence of Au-NP in the final structure, XRD analysis was done (Figure 6). The XRD 


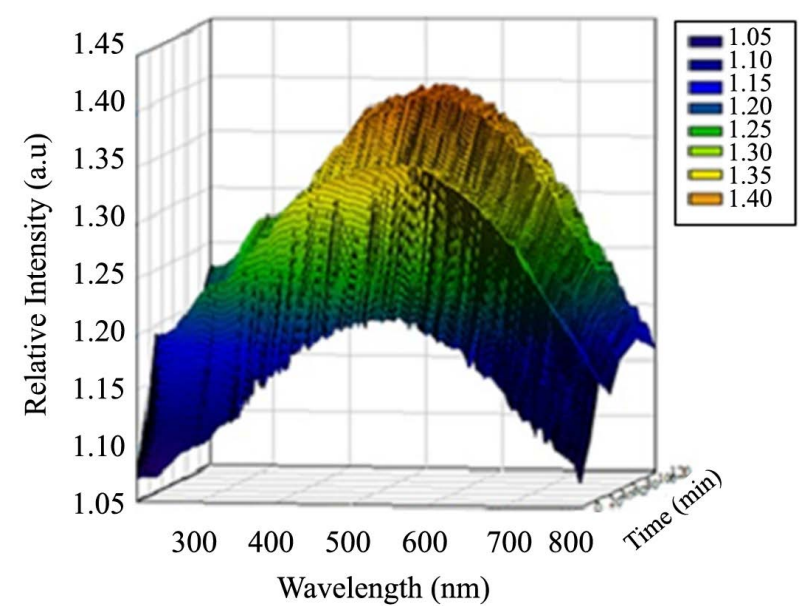

Figure 2. UV-vis spectra of dendrimer-gold complex during the synthesis.

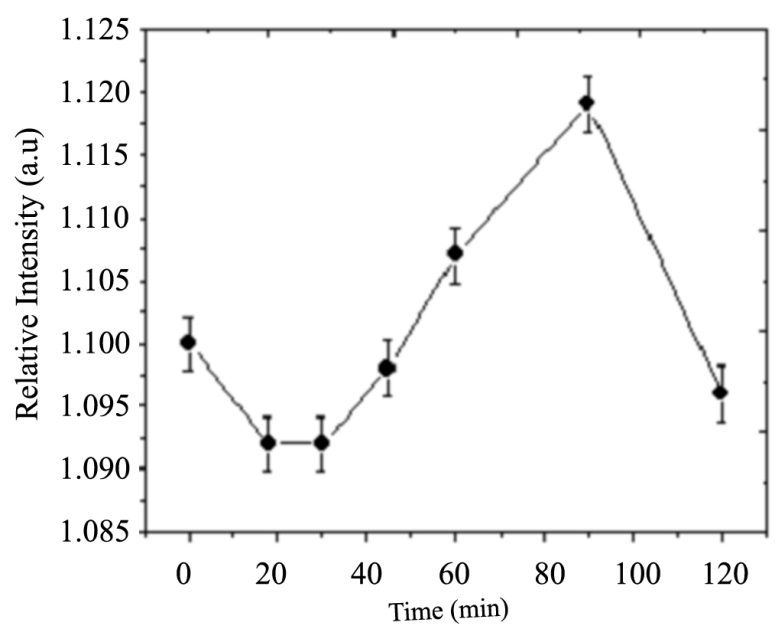

Figure 3. Change of intensity ratio of $520 \mathrm{~nm}$ to $280 \mathrm{~nm}$ peaks during synthesis of magnetodendimer-gold nanocomposite.

patterns contain diffraction peaks at $2 \theta$ equal to 38, 44.3, 64.5 and 77.9 which represent (111), (200), (220) and (311) crystallographic plane respectively which prove the FCC structure of gold (JCPDS No.00-04-0784) [26] [44]-[46]. The broadening of the most intense peak of these nanoparticles demonstrates that the small size Au-NPs could be calculated by using the Scherrer equation (Equation (1)) [47]. The calculation shows that the average particle size of Au-NPs obtained using $\mathrm{NaBH}_{4}$ reducing agent is about 4 orders larger than those formed by hydrazine sulfate.

$$
D=\frac{K \lambda}{\beta \cos \theta}
$$

where, $D, \lambda$ and $\beta$ represent the mean diameter of particles, the wavelength of incident $\mathrm{X}$-ray, and the full width at half height (FWHM) respectively and constant $K$ is equal to 0.9 .

\subsection{FTIR and WDX Evaluation}

FTIR analysis was used to prove the attachment of Au-NPs to magnetodendrimer shown in Figure 7. In the dendrimer-modified iron oxide nanoparticle (ID), the amide bands at 1570 and $1630 \mathrm{~cm}^{-1}$ represent the characteristics of PAMAM dendrimer. Esumi et al. believe that the interactions of Au-NPs with amine terminated den- 


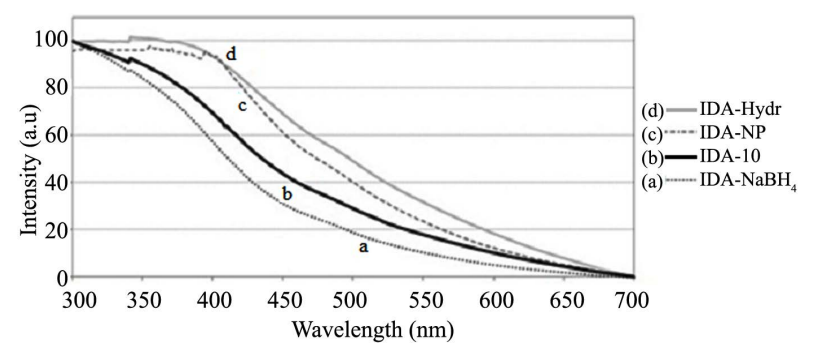

Figure 4. UV-vis spectra of magnetodendimer-gold nanocomposite a) IDA, b) IDA 10, c) IDA NP, d) IDA Hydr.

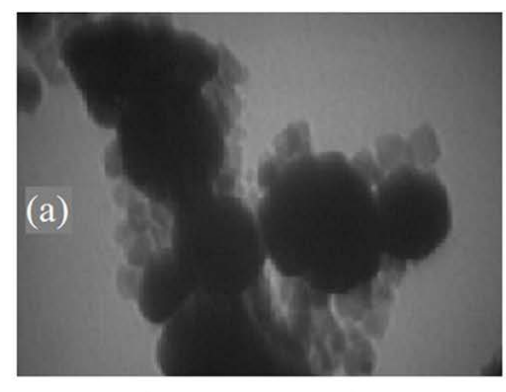

$100 \mathrm{~nm}$
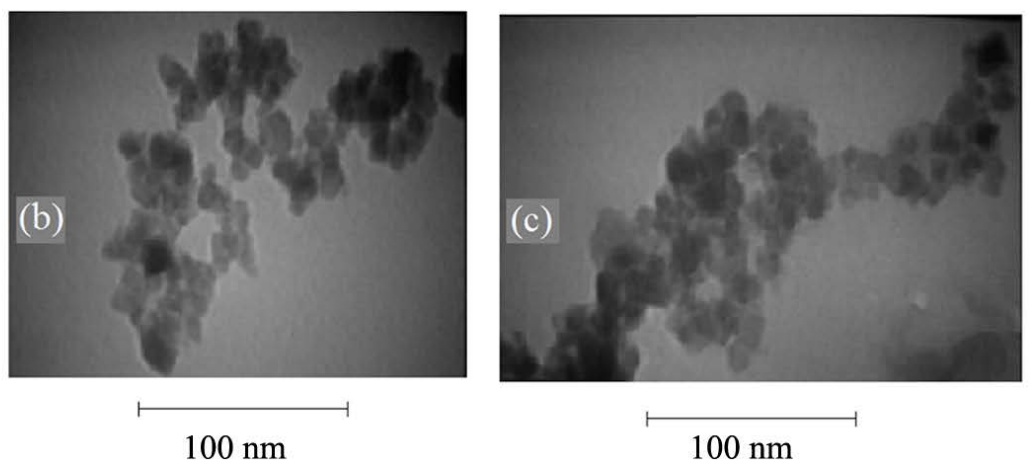

Figure 5. TEM image of a) IDA-10; b) IDA- Hydr and c) IDA-NP.

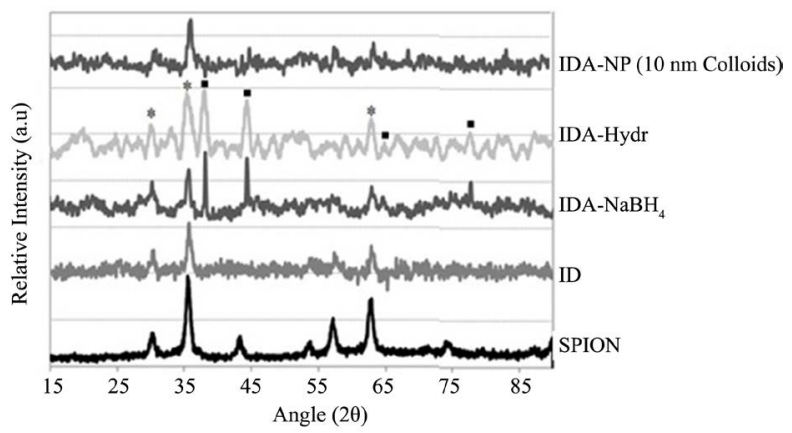

Figure 6. XRD pattern of a) SPION; b) ID; c) IDA-NaBH ${ }_{4}$; d) IDA-Hydr; e) IDA-NP ( ${ }^{*}$ represents magnetite phase and $\boldsymbol{m}$ indicates gold phase).

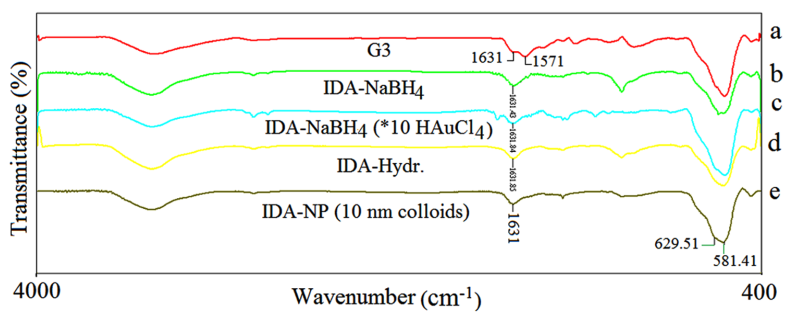

Figure 7. FTIR Spectra of a) G 3; b) IDA- $\mathrm{NaBH}_{4}$; c) IDA-10; d) IDA-Hydr; e) IDA-NP.

drimers are stronger than carboxyl terminated dendrimers as the FTIR bands of dendrimer are shifted while the half generations do not show any changes [10] [48]. Furthermore, according to [34] the FTIR and UV-vis evaluations of dendrimer depend on the size and their generations, at lower generations' external type and at higher 
generations a mixed type could be observed.

Type I amide band (wave number of $1630 \mathrm{~cm}^{-1}$ ) is generally related to $\mathrm{C}=\mathrm{O}$ stretching vibration $(70 \%-85 \%)$ and directly appertain to combination and structure of polymer backbone. Type II amide band (wave number $1570 \mathrm{~cm}^{-1}$ ) represents the N-H bending vibration (20\%) [49]-[52]. As shown in Figure 6, FTIR spectra regarding IDA complex samples do not contain the band at $1570 \mathrm{~cm}^{-1}$ and the band $1630 \mathrm{~cm}^{-1}$ remain without any changes. This shows that the Au-NPs interact with N-H groups of MD structure whereas the polymer backbone relatively retains its original structure. In this figure, the peaks at 581 and $629 \mathrm{~cm}^{-1}$ represent the iron oxide phase at all synthesized structures. In order to quantitatively appraise attachment of Au-NPs to MD, WDX (Wavelength-dispersive X-ray spectroscopy) analysis was performed. The analysis confirms the presence of gold phase in the prepared nanoparticles (Figure 8) [46] and Figure 9 shows the efficiency of synthesis in terms

(L)

(a)

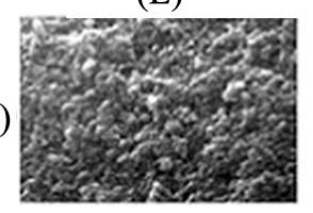

(b)

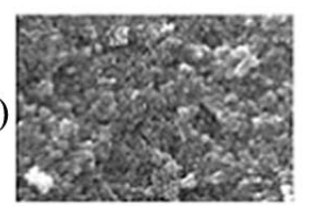

(c)

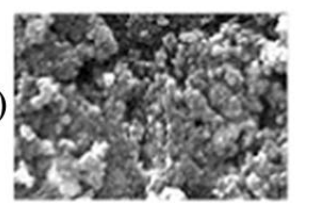

(d)

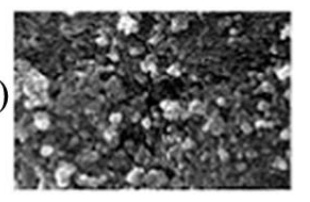

(M)
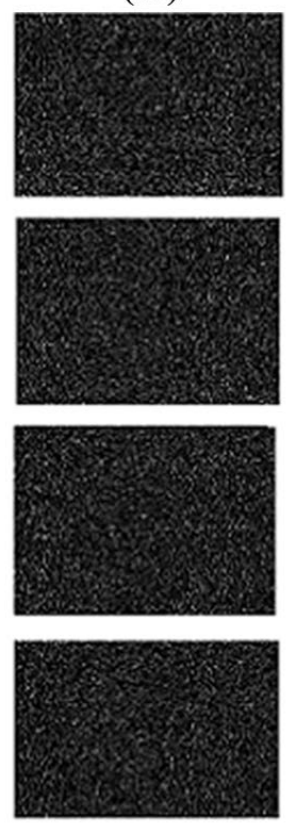

$500 \mathrm{~nm}$
(R)
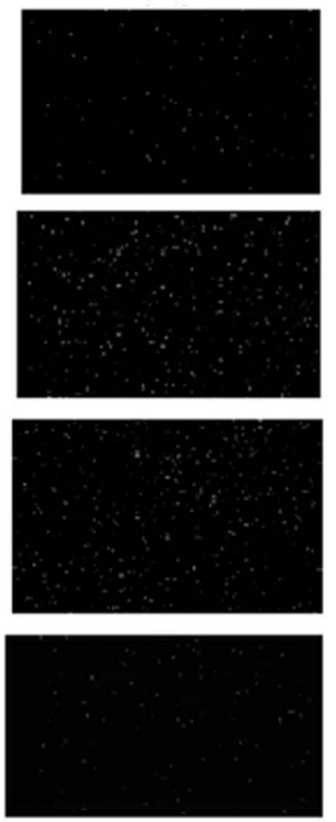

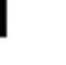

Figure 8. WDX images of a) IDA- $\mathrm{NaBH}_{4}$; b) IDA-10; c) IDA-Hydr; d) IDANP nanoparticles at three different levels of synthesized nanoparticles (L), Fe element (M), and Au element (R) respectively.

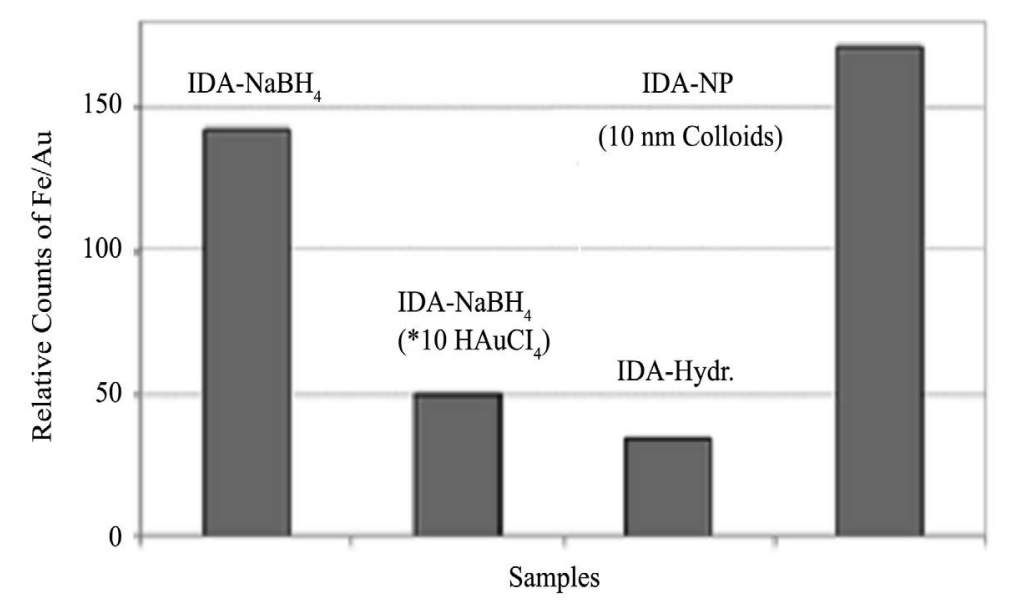

Figure 9. Relative Counts of $\mathrm{Fe} / \mathrm{Au}$ for different synthesized nanoparticles. 
of relative counts of Fe: Au where hydrazine sulfate reducing agent has shown to produce the best nanoparticles compared to other synthesis methods.

\subsection{Laser-Induced Fluorescence Spectroscopy}

One of the early works regarding the fluorescence of PAMAM dendrimer was reported by Klajnart et al. [53]. It has been shown that after addition of $\mathrm{HAuCL}_{4}$, the fluorescence intensity is red shifted towards the longer wavelength [6] [36]. After adding the reducing agent, the peaks are changed considerably which shows the close attachment of Au-NPs to the dendrimers. The shift of fluorescence spectra could be explained by strong interaction between gold crystals and amine groups of dendrimer [35]. Optical properties of Au-NPs are highly sensitive to their particle size and have an effective influence on the fluorescence spectra of dendrimer [39] [40]. This is because of the internal interaction between flexible structure of dendrimer and rigid structure of entrapped Au-NPs, whereas the larger particles damp the fluorescence of dendrimers. These phenomena could be explained in terms of fluorescence resonance energy transfer (FRET) [40]. In this study, IDA-Hydr nanocomposites strongly changed the initial fluorescent spectra of MD, i.e. the shape and location of peaks were sharply changed. In most cases, the fluorescence spectra of IDA-Hydr are stronger than others with highest signals at about $525 \mathrm{~nm}$ and $550 \mathrm{~nm}$ respectively. One reason for this is more likely due to their smaller size which helps them to be entrapped easily by dendrimers and hence intensify the fluorescence signals, see Figure 10.

Considering the chemical structures of collagen and cotton, there is a possibility of hydrogen interaction between the hydroxyl group of the substrate and amine group of dendrimer nanocomposites which produces a new fluorescent moieties with a time dependent behaviour. The longer the time, the more fluorescent moieties is produced hence, more intense fluorescence image could be obtained. Figure 11 and Figure 12 represent the fluorescence image of nanoparticles with different formulation in close contact with collagen and cotton, respectively.

\subsection{Thermal Response of Synthesized Nanoparticles}

Figure 13 shows the thermal response of different synthesized nanocomposites at $488 \mathrm{~nm}$ (Figure 13(a)) and

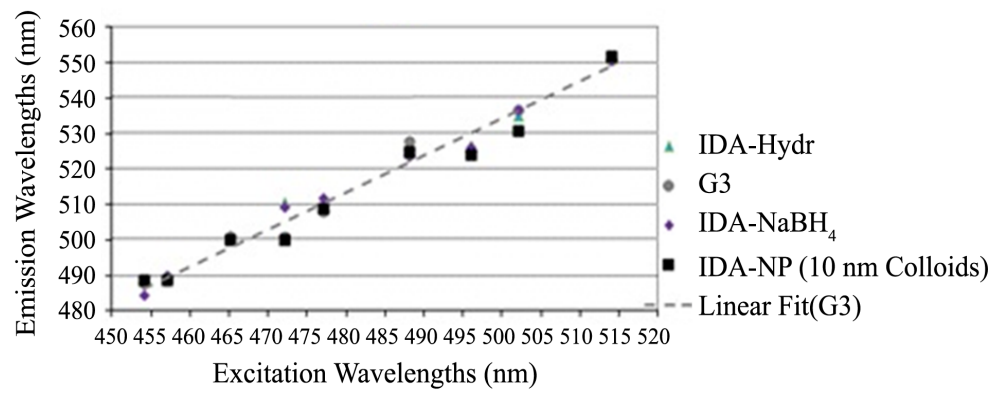

(a)

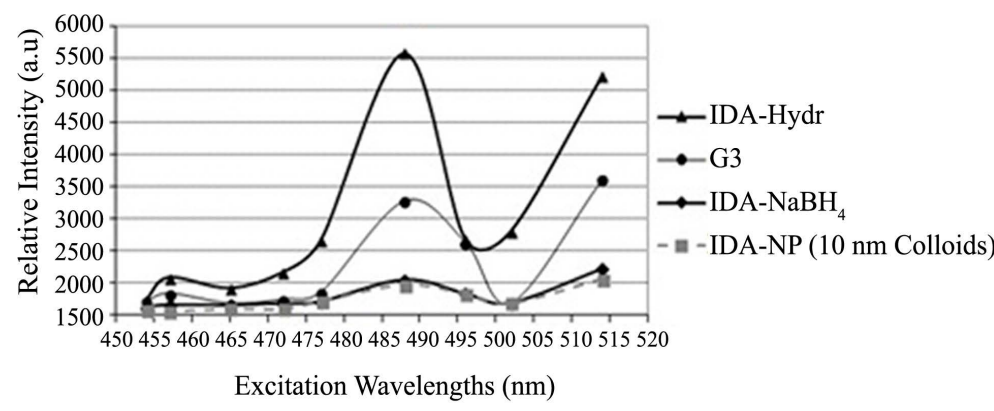

(b)

Figure 10. Fluorescence of emission wavelengths (a) and the relative intensities (b) of G3, IDA-NP, IDA-10, IDA-Hydr, IDA- $\mathrm{NaBH}_{4}$ at different excitation wavelengths. 
(a)
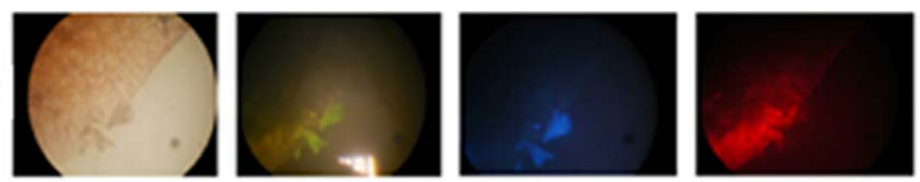

(b)
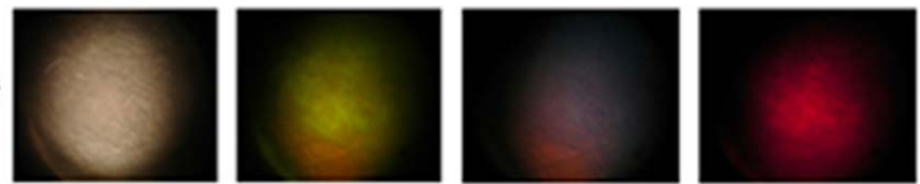

(c)
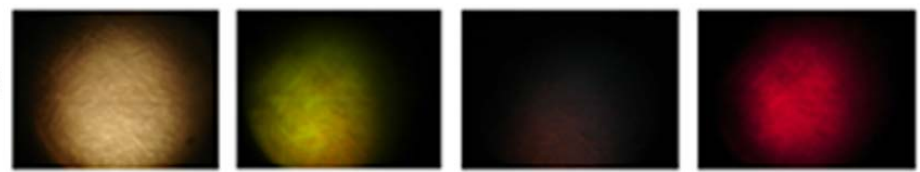

(d)
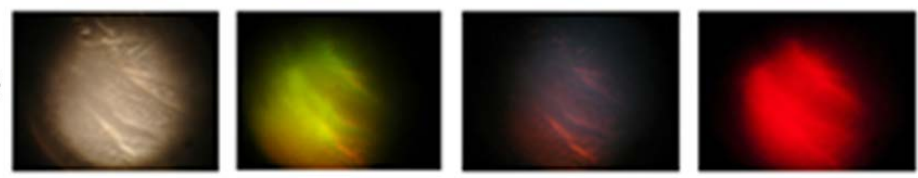

(e)
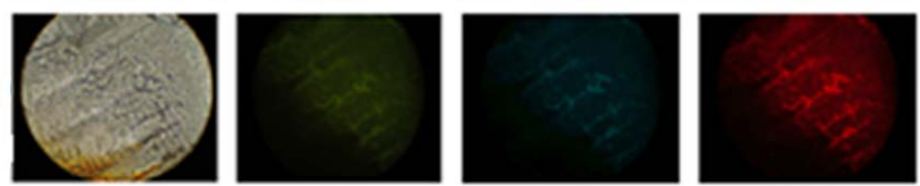

Figure 11. Fluorescence image of a) G3; b) IDA-NaBH 4 ; c) IDA-10; d) IDAHydr; and e) IDA-NP nanoparticles on the collagen substrate.

(a)
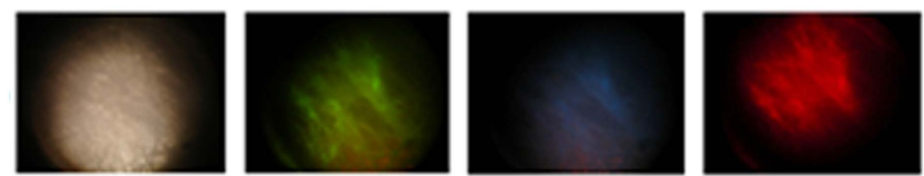

(b)
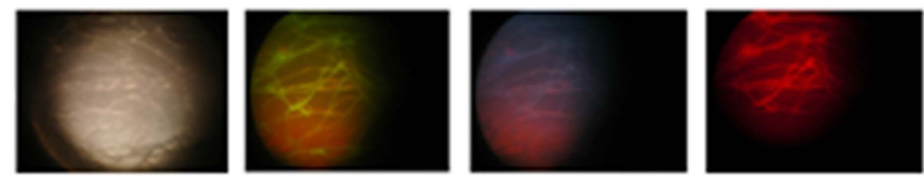

(c)
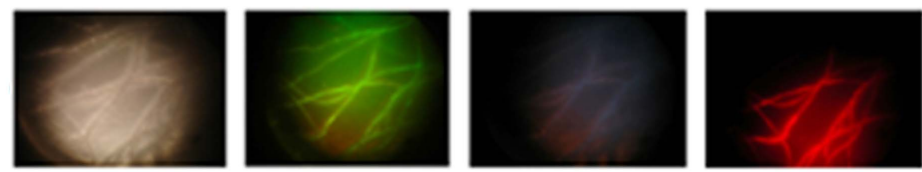

(d)
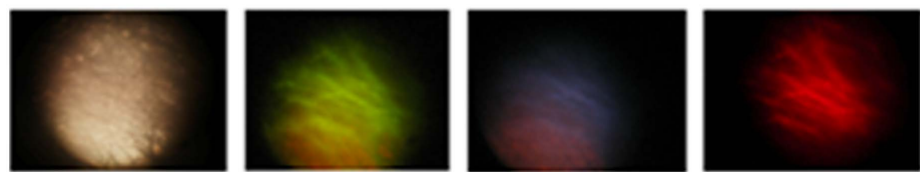

(e)
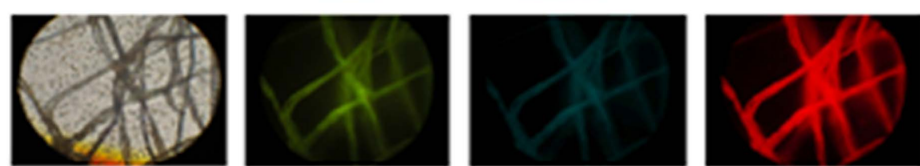

Figure 12. Fluorescence image of a) G3; b) IDA-NaBH ; c) IDA-10; d) IDAHydr; and e) IDA-NP nanoparticles on the cotton substrate.

$514 \mathrm{~nm}$ (Figure 13(b)) for 5 minutes. Clearly, the highest temperature rise was produced by IDA-NaBH4 at 488 $\mathrm{nm}$ and pure Au-NPs at $514 \mathrm{~nm}$ due to its proximity to gold surface plasmon resonance (SPR) peak absorption at about $530 \mathrm{~nm}$. It has been reported hat photon excitation of metallic nanostructures leads to production of hot 


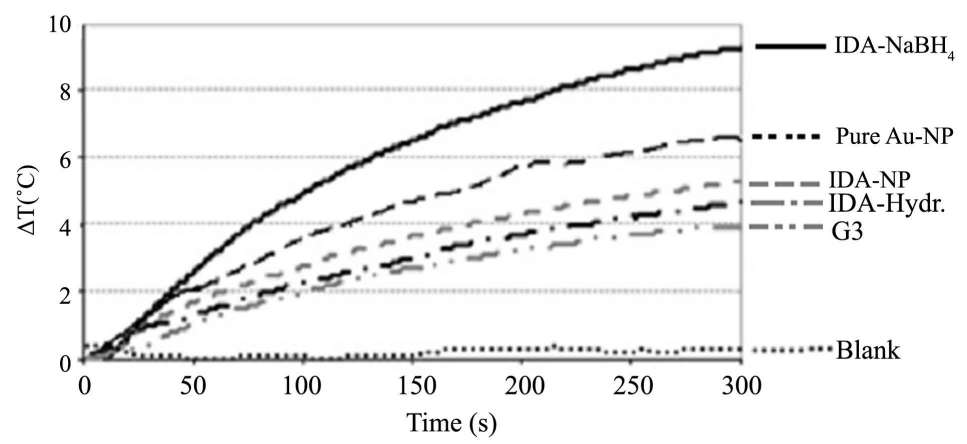

(a)

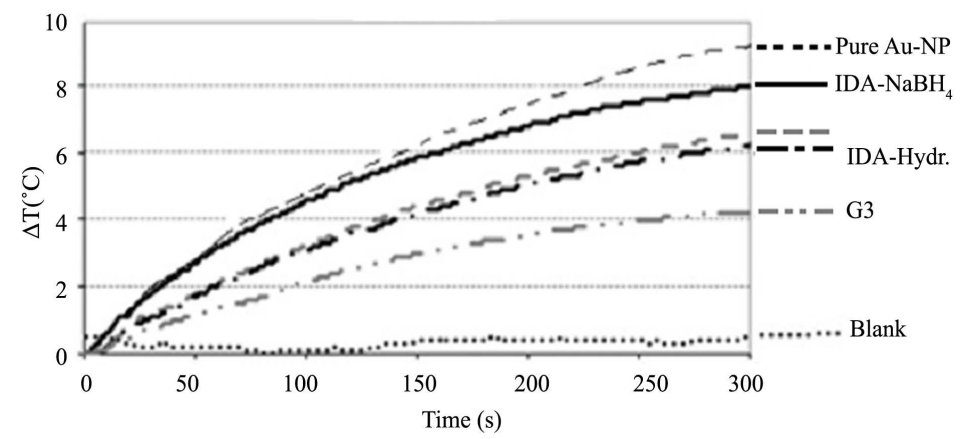

(b)

Figure 13. The effect of laser irradiation at two different wavelengths of a) $488 \mathrm{~nm}$ and b) $514 \mathrm{~nm}$ on thermal behaviour of different synthesized nanoparticles.

electron cloud which cools down in about 1 ps as a result of heat transfer within the nanoparticle lattice which is then followed by phonon-phonon interactions in 100 ps where the metallic lattice transfers the heat to its surrounding medium and cools down [54]-[59]. The phenomena of energy absorption and increasing the temperature are well known in SPR frequencies where these rapid changes of temperature lead to variety of applications of nanocomposites.

The temperature distribution around optically-stimulated plasmonic nanoparticles can be described by the following equation [60]:

$$
\rho c \frac{\partial T}{\partial t}=\nabla \cdot(K \nabla T)+\langle p\rangle
$$

where $T$ is the local temperature, and $\rho, c$, and $K$ represent density, specific heat capacity, and thermal conductivity of material, respectively. Here $\langle p\rangle$ is the average heat power dissipated inside the NP and the heat power is given by $P=\langle p\rangle$. $V_{N P}$ indicated using Equation 3:

$$
P=4 \pi R^{3} K_{A u} \frac{C \varepsilon_{w}}{2}|E|^{2} \operatorname{Im}\left(\frac{\varepsilon_{A u}-\varepsilon_{w}}{\varepsilon_{A u}+2 \varepsilon_{w}}\right)=4 \pi R^{3} K_{A u} \frac{C \varepsilon_{w}}{2}\left|E_{0}\right|^{2}\left|\frac{3 \varepsilon_{w}}{\varepsilon_{A u}+2 \varepsilon_{w}}\right|^{2} \operatorname{Im}\left(\frac{\varepsilon_{A u}-\varepsilon_{w}}{\varepsilon_{A u}+2 \varepsilon_{w}}\right)
$$

where $C$ is the speed of light, $\varepsilon_{A u}$ and $\varepsilon_{w}$ are the permittivity of gold and water, respectively. This definition obtained from the original formula [61]:

$$
P=\sigma_{a b s} I
$$

$\sigma_{a b s}$ is the optical absorption cross section of the gold nanoparticles and I is the intensity of incoming light.

$$
I=\frac{C \varepsilon_{w}}{2}|E|^{2}
$$


And

$$
E=E_{0} \frac{3 \varepsilon_{w}}{\varepsilon_{A u}+2 \varepsilon_{w}}
$$

After a transient evolution, materials reach steady state temperature profile under CW illumination. Dimensional analysis of the two diffusion equations demonstrates two time scale of the system:

$$
\begin{aligned}
& \tau_{d}^{w}=\frac{\rho_{w} c_{w}}{K_{w}} R^{2}=\frac{R^{2}}{k_{w}} \\
& \tau_{d}^{A u}=\frac{\rho_{A u} c_{A u}}{K_{A u}} R^{2}=\frac{R^{2}}{k_{A u}}
\end{aligned}
$$

where $k=K / \rho c$ is thermal diffusivity $\left(\mathrm{m}^{2} \cdot \mathrm{s}^{-1}\right), \tau_{d}^{w}$ and $\tau_{d}^{A u}$ represent the characteristic times associated with the evolution of the temperature profile in the surrounding water and inside Au-NP, respectively. Thermalization inside the nanoparticle happens much faster than the water, as $k_{A u}$ is far greater than $k_{w}$. Hence, the best consideration of reaching comprehensive temperature profile of the total system is directed by the time scale $\tau_{d}^{w}$ [61].

In the final steady state regime, Equation (2) reduces to:

$$
\nabla \cdot(K \nabla T(r))=-\langle p\rangle(r)
$$

This equation is formally equivalent to Poisson's equation and produces a profile of temperature increase $\Delta T$ given by a Coulomb potential outside the particle [8] [58] [62].

$$
\Delta T(r)=\frac{P}{4 \pi K_{w} r} \text { for } r \geq R
$$

Substituting the Equation (3) in Equation (10), it gives the temperature increase at the surface of nanoparticle (i.e. $r=R$ ),

$$
\Delta T(r)=\frac{I K_{A u} R^{2}}{K_{w}} \operatorname{Im}\left(\frac{\varepsilon_{A u}-\varepsilon_{w}}{\varepsilon_{A u}+2 \varepsilon_{w}}\right) \approx 2.00 \times \frac{I K_{A u} R^{2}}{K_{w}}
$$

here $R$ represents the nanoparticle's radius and $\mathrm{I}$ is the intensity of incident beam. Equation (11) shows that temperature of the system containing plasmonic nanoparticles is proportional to the square of the nanoparticle radius, i.e. $\Delta T \propto R^{2}$. As the TEM results show, the use of sodium borohydrate as a reducing agent produced clusters of Au-NPs which consists a greater particle size than the other formulation. Based on above analysis we expect to obtain a higher temperature in IDA- $\mathrm{NaBH}_{4}$ shown in Figure 13. Also this figure indicates that IDA-Hydr formulation leads to the least significant changes in temperature which could be described by its smaller particles size compare to other gold containing formulations. Furthermore, it was shown that temperature of NP changes with regard to their exciting wavelengths and hence the maximum spectral position of the plasmonic band [49] [58] [60] i.e., the temperature drastically decreases at both lower and higher wavelengths. As IDA nanoparticles contain clusters, their plasmonic peak may be obtained at $320 \mathrm{~nm}$. The thermal analysis indicates that at shorter exciting wavelengths, these nanocomposites produce more heat than the longer wavelengths and on the other hand longer wavelengths cause more temperature increase in the case of pure Au-NPs. This lies on the fact that the shorter exciting wavelength $(488 \mathrm{~nm})$ is closer to the plasmonic band of the Au-NPs than $514 \mathrm{~nm}$.

\section{Conclusion}

Gold-magneto dendrimer nanocomposites were prepared using two different reducing agents and pre-synthesized Au-NP. To the best of our knowledge, there was no evidence of the in-situ monitoring of the Au-NP formation which was done in this study. WDX, XRD, and FTIR analysis of the nanocomposites indicated the simultaneous presence of magnetite and gold phase in the materials. Also, the WDX and TEM results suggest that the use of hydrazine sulfate as a reducing agent can both produce a uniform structure and load higher degree of $\mathrm{Au}-\mathrm{NPs}$ in the materials. LIF spectroscopy and fluorescent microscopy results confirmed that synthesized met- 
al/dendrimer nanocomposites could fluorescently dye biopolymers such as cotton and collagen. The interaction of $488 \mathrm{~nm}$ and $514 \mathrm{~nm}$ wavelengths with the all three nanocomposite media resulted in temperature increase with IDA- $\mathrm{NaBH}_{4}$ and IDA-Hydr showing the highest and the lowest temperature at $488 \mathrm{~nm}$ respectively. At 514 $\mathrm{nm}$, however, pure-Au NP synthesized by hydrazine sulfate exhibited the highest heating effect which was closer to its maximum absorption peak of plasmonic band. Considering the outcomes of these experiments, we suggest that these novel nanocomposites can be employed as biomarker and therapeutic agents based on their magnetic, fluorescence and SPR-based optothermal properties.

\section{References}

[1] Pankhurst, Q., Connolly, J., Jones, S. and Dobson, J. (2003) Applications of Magnetic Nanoparticles in Biomedicine. Journal Physics D: Applied Physics, 36, 167-181. http://dx.doi.org/10.1088/0022-3727/36/13/201

[2] Berry, C. and Curties, A. (2003) Functionalization of Magnetic Nanoparticles for Applications in Biomedicine. Journal Physics D: Applied Physics, 36, 198-206. http://dx.doi.org/10.1088/0022-3727/36/13/203

[3] Khosroshahi, M.E. and Nourbakhsh, M. (2011) In Vitro Skin Wound Soldering Using $\mathrm{SiO}_{2} / \mathrm{Au}$ Nanoshells and a Diode Laser. Medical Laser Application, 26, 35-42. http://dx.doi.org/10.1016/j.mla.2010.06.001

[4] Wang, Y., Xie, X. and Goodson, T. (2005) Enhanced Third-Order Nonlinear Optical Properties in Dendrimer-Metal Nanocomposites. Nano Letter, 5, 2379-2384. http://dx.doi.org/10.1021/nl051402d

[5] Shi, X., Wang, S., Shen, M., Antwerp, M. and Chen, X. (2009) Multifunctional Dendrimer-Modified Multiwalled Carbon Nanotubes: Synthesis, Characterization, and in Vitro Cancer Cell Targeting and Imaging. Biomacromolecules, 10, 1744-1750. http://dx.doi.org/10.1021/bm9001624

[6] Stemmler, M., Stefani, F., Bernhardt, S., Bauer, R. and Kreiter M. (2009) One-Pot Preparation of Dendrimer-Gold Nanoparticle Hybrids in a Dipolar Aprotic Solvent. Langmuir, 25, 12425-12428. http://dx.doi.org/10.1021/la902354e

[7] Thompson, D., Hermes, J., Quinn, A. and Mayor, M. (2012) Scanning the Potential Energy Surface for Synthesis of Dendrimer-Wrapped Gold Clusters: Design Rules for True Single-Molecule Nanostructures. ACS Nano, 6, 3007-3017. http://dx.doi.org/10.1021/nn204470g

[8] Baffou, G. and Quidan, R. (2013) Thermo-Plasmonics: Using Metallic Nanostructures as Nano-Sources of Heat. Laser \& Photonics Reviews, 7, 171-187. http://dx.doi.org/10.1002/lpor.201200003

[9] Hassannejad, Z. and Khosroshahi, M.E. (2013) Synthesis and Evaluation of Time Dependent Optical Properties of Plasmonic-Magnetic Nanoparticles. Optical Materials, 35, 644-651. http://dx.doi.org/10.1016/j.optmat.2012.10.019

[10] Esumi, K., Houdatsu, H. and Yoshimur, T. (2004) Antioxidant Action by Gold-PAMAM Dendrimer Nanocomposites. Langmuir, 20, 2536-2538. http://dx.doi.org/10.1021/la036299r

[11] Wang, X., Haung, Sh., Shan, Z. and Yang, W. (2009) Preparation of $\mathrm{Fe}_{3} \mathrm{O}_{4} @ \mathrm{Au}$ Nano-Composites by Self-Assembly Technique for Immobilization of Glucose Oxidase. Chinese Science Bulletin, 54, 1176-1181. http://dx.doi.org/10.1007/s11434-009-0113-7

[12] Khosroshahi, M. E. and Ghazanfar, L. (2012) Physicochemical Characterization of $\mathrm{Fe}_{3} \mathrm{O}_{4} / \mathrm{SiO}_{2} /$ Au Multilayer Nanostructure. Materials Chemistry Physics, 133, 55-62. http://dx.doi.org/10.1016/j.matchemphys.2011.12.047

[13] Shi, X., Sun, K. and Baker Jr., J.R. (2009) Spontaneous Formation of Functionalized Dendrimer-Stabilized Gold Nanoparticles. The Journal of Physical Chemistry C, 112, 8251-8258. http://dx.doi.org/10.1021/jp801293a

[14] Shen, M. and Shi, X. (2010) Dendrimer-Based on Organic/Inorganic Hybrid Nanoparticles in Biomedical Applications. Nanoscale, 2, 1596-1610. http://dx.doi.org/10.1039/c0nr00072h

[15] Majoros, I., Myc, A., Thomas, T., Mehta, C. and Baker, J. (2006) PAMAM Dendrimer-Based Multifunctional Conjugate for Cancer Therapy: Synthesis, Characterization, and Functionality. Biomacromolecules, 7, 572-579. http://dx.doi.org/10.1021/bm0506142

[16] Syahir, A., Tomizaki, K., Kajikawa, K. and Mihara, H. (2009) Poly(Amidoamine)-Dendrimer Modified Gold Surfaces for Anomalous Reflection of Gold to Detect Biomolecular Interactions. Langmuir, 25, 3667-3674. http://dx.doi.org/10.1021/la8028275

[17] Choi, S., Myc, A., Silpe, J., Sumit, M., Wong, P. and McCarthy, K. (2012) Dendrimer-Based Multivalent Vancomycin Nanoplatform for Targeting the Drug-Resistant Bacterial Surface. ACS Nano, 7, 214-228. http://dx.doi.org/10.1021/nn3038995

[18] Al-Jamal, K., Al-Jamal, W., Wang, J., Rubio, N. and Buddle, J. (2013) Cationic Poly-L-Lysine Dendrimer Complexes Doxorubicin and Delays Tumor Growth In-vitro and In-Vivo, ACS Nano,7, 1905-1917. http://dx.doi.org/10.1021/nn305860k

[19] Venditto, V., Regino, C. A. and Brechbiel, M. (2005) PAMAM Dendrimer Based Macromolecules as Improved Con- 
trast Agents. Molecular Pharmacutics, 2, 302-311. http://dx.doi.org/10.1021/mp050019e

[20] Bronstein, L. and Shifrina, L. (2011) Dendrimers as Encapsulating, Stabilizing, or Directing Agents for Inorganic Nanoparticles. Chemical Reviews, 111, 5301-5344. http://dx.doi.org/10.1021/cr2000724

[21] Garcia, M. and Baker, L. (1998) Crooks Preparation and Characterization of Dendrimer-Gold Colloid Nanocomposites. Analytical Chemistry, 71, 256-258. http://dx.doi.org/10.1021/ac980588g

[22] Esumi, K., Matsumoto, T., Seto, Y. and Yoshimura,T. (2005) Preparation of Gold-Gold/Silver-Dendrimer Nanocomposites in the Presence of Benzoin in Ethanol by UV Irradiation. Journal Colloid Interface Science, 284, 199-203. http://dx.doi.org/10.1016/j.jcis.2004.09.020

[23] Hoffman, L.W., Andersson, G., Sharma, A., Clarke, S. and Voelcker, N. (2011) New Insigh into the Structure of PAMAM Dendrimer/Gold Nanoparticle Nanocomposites. Langmuir, 27, 6759-6767. http://dx.doi.org/10.1021/la1050964

[24] Sardar, R., Funston, A., Mulvaney, P. and Murray, R. (2009) Gold Nanoparticles: Past, Present, and Future. Langmuir, 25, 13840-13851. http://dx.doi.org/10.1021/la9019475

[25] Li, Z., Huang, P., Zhang, X., Lin, J., Yang, S. and Liu, B. (2009) RGD-Conjugated Dendrimer-Modified Gold Nanorods for in Vivo Tumor Targeting and Photothermal Pherapy. Molecular Pharmaceutics, 7, 94-104. http://dx.doi.org/10.1021/mp9001415

[26] Khosroshahi, M.E. and Nourbakhsh, M. (2010) Preparation and Characterization of Self Assembled Gold Nanoparticles on Amino Functionalized $\mathrm{SiO}_{2}$ Dielectric Core. World Academy of Science, Engineering and Technology, 64, 353-356.

[27] Tajabadi, M. and Khosroshahi, M.E. (2012) Effect of Alkaline Media Concentration and Modification of Temperature on Magnetite Synthesis Method Using $\mathrm{FeSO}_{4} / \mathrm{NH}_{4} \mathrm{OH}$. International Journal Chemical Engineering Applications, 3, 206-210. http://dx.doi.org/10.7763/IJCEA.2012.V3.187

[28] Tajabadi, M., Khosroshahi, M.E. and Bonakdar, S. (2013) An Efficient method of SPION Synthesis Coated With Third Generation PAMAM Dendrimer, Colloids Surface A: Physicochem Engineering Aspects, 431, 18-26. http://dx.doi.org/10.1016/j.colsurfa.2013.04.003

[29] Okugaichi, A., Torigoe, K., Yoshimura, T. and Esumi, K. (2006) Interaction of Cationic Gold Nanoparticles and Carboxylate-Terminated Poly(Amidoamine) Dendrimers. Colloids and Surfaces A: Physicochem Engineering Aspects, 273, 154-160. http://dx.doi.org/10.1016/j.colsurfa.2005.08.028

[30] Balogh, L., Valluzzi, R., Laverdure, K., Gido, S., Hagnauer, G. and Tomalia, D. (1999) Formation of Silver and Gold Dendrimer Nanocomposites. Journal Nanoparticle Research, 1, 353-368. http://dx.doi.org/10.1023/A:1010060404024

[31] He, J., Valluzzi, R., Yang, K., Dolukhanyan, T., Sung, C., Kumar, J. and Tripathy J. (1999) Electrostatic Multilayer Deposition of a Gold-Dendrimer Nanocomposite. Chemisrty of Materials, 11, 3268-3274. http://dx.doi.org/10.1021/cm990311c

[32] Balogh, L. and Tomalia, D. (1998) Poly(Amidoamine) Dendrimer-Templated Nanocomposites. Synthesis of Zero Valent Copper Nanoclusters. Journal of American Chemical Society, 120, 7355-7356. http://dx.doi.org/10.1021/ja980861w

[33] Zhao, M., Sun, L. and Crooks, R. (1998) Preparation of Cu Nanoclusters within Dendrimer Templates. Journal of American Chemical Society, 120, 4877-4878. http://dx.doi.org/10.1021/ja980438n

[34] Satoh, K., Yoshimura, T. and Esumi, K. (2002) Effects of Various Thiol Molecules Added on Morphology of Dendrimer-Gold Nanocomposites. Journal of Colloid Interface Science, 255, 312-322. http://dx.doi.org/10.1006/jcis.2002.8585

[35] Shi, X., Ganser, T., Sun, K. and Baker Jr., J.R. (2006) Characterization of Crystalline Dendrimer-Stabilized Gold Nanoparticles. Nanotechnology, 17, 1072-1078. http://dx.doi.org/10.1088/0957-4484/17/4/038

[36] Kim, Y., Oh, S. and Crooks,R. (2003) Preparation and Characterization of 1 - 2 nm Dendrimer-Encapsulated Gold Nanoparticles Having Very Narrow Size Distributions. Chemistry of Materials, 16, 167-172. http://dx.doi.org/10.1021/cm0349320

[37] Mosseri, S., Henglein, A. and Janata, E. (1989) Reduction of Dicyanoaurate(I) in Aqueous Solution: Formation of Nonmetallic Clusters and Colloidal Gold. Journal of Physical Chemistry, 93, 6791-6795. http://dx.doi.org/10.1021/j100355a042

[38] Divsar, F., Nomani, A., Chaloosi, M. and Haririan, I. (2009) Synthesis and Characterization of Gold Nanocomposites with Modified and Intact Polyamidoamine Dendrimers. Microchimica Acta, 165, 421-426. http://dx.doi.org/10.1007/s00604-009-0156-0

[39] Jiang, G., Wang, Y. and Sun, X. (2010) Fluence on Fluorescence Properties of Hyperbranched Poly(Amidoamine)s by Nano Golds. Journal of Polymer Science Polymer Physics, 48, 2386-2391. http://dx.doi.org/10.1002/polb.22137 
[40] Zhang, Z., Rong, F., Niu, S., Xie, Y., Wang, Y., Yang, H. and Fu, D. (2010) Investigation the Effects of Nano Golds on the Fluorescence Properties of the Sectorial Poly(Amidoamine) (PAMAM) Dendrimers. Applied Surface Science, 256, 7194-7199. http://dx.doi.org/10.1016/j.apsusc.2010.05.049

[41] Westcott, S., Oldenburg, S., Lee, T. and Halas, N. (1998) Formation and Adsorption of Clusters of Gold Nanoparticles onto Functionalized Silica Nanoparticle Surfaces. Langmuir, 14, 5396-5401. http://dx.doi.org/10.1021/la980380q

[42] Zheng, J., Petty, J. and Dickson, R. (2003) High Quantum Yield Blue Emission from Water-Soluble Au-Nanodots. Journal of American Chemical Society, 125, 7780-7781. http://dx.doi.org/10.1021/ja035473v

[43] Torigoe, K., Suzuki, A. and Esumi, K. (2001) Au(III)-PAMAM Interaction and Formation of Au-PAMAM Nanocomposites in Ethyl Acetate. Journal of Colloid Interface Science, 241, 346-356. http://dx.doi.org/10.1006/jcis.2001.7778

[44] Arias, J., Gallardo, V., Gómez-Lopera, S., Plaza, R. and Delgado, A. (2001) Synthesis and Characterization of Poly(ethyl-2-Cyanoacrylate) Nanoparticles With a Magnetic Core. Journal of Control Release, 77, 309-321. http://dx.doi.org/10.1016/S0168-3659(01)00519-3

[45] Wu, C., Yu, C. and Chu, M. (2011) A Gold Nanoshell with a Silica Inner Shell Synthesized Using Liposome Templates for Doxorubicin Loading and Near-Infrared Photothermal Therapy. International Journal of Nanomedicine, 6 , 807-813.

[46] Streszewski, B., Jaworski, W., Pacławski, K., Csapó E, Dékány, I. and Fitzner, K. (2012) Gold Nanoparticles Formation in the Aqueous System of Gold(III) Chloride Complex Ions and Hydrazine Sulfate-Kinetic Studies. Colloids Surface A: Physicochemical Engineering Aspects, 397, 63-72. http://dx.doi.org/10.1016/j.colsurfa.2012.01.031

[47] Faiyas, A., Vinod, E., Joseph, J., Ganesan, R. and Pandey, R. (2009) Dependence of pH and Surfactant Effect in the Synthesis of Magnetite $\left(\mathrm{Fe}_{3} \mathrm{O}_{4}\right)$ Nanoparticles and Its Properties. Journal of Magnetism and Magnetic Materials, 322, 400-404. http://dx.doi.org/10.1016/j.jmmm.2009.09.064

[48] Esumi, K., Suzuki, A., Yamahira, A. and Torigoe, K. (2000) Role of Poly(Amidoamine) Dendrimers for Preparing Nanoparticles of Gold, Platinum, and Silver. Langmuir, 16, 2604-2608. http://dx.doi.org/10.1021/la991291w

[49] Grabchev, I., Bojinov, V. and Chovelon, J. M. (2003) Synthesis, Photophysical and Photochemical Properties of Fluorescent Poly(Amidoamine) Dendrimers. Polymer, 44, 4421-4428. http://dx.doi.org/10.1016/S0032-3861(03)00407-5

[50] Li, J., Chen, Q. and Yang, L. (2010) The Synthesis of Dendrimer Based on the Dielectric Barrier Discharge Plasma Grafting Amino Group Film. Surface Coating Technology, 205, S257-S260. http://dx.doi.org/10.1016/j.surfcoat.2010.07.083

[51] Chou, C. and Lien, H. (2009) Dendrimer-Conjugated Magnetic Nanoparticles for Removal of Zinc (II) From Aqueous Solutions. Journal of Nanoparticles Resesearch, 13, 2099-2107. http://dx.doi.org/10.1007/s11051-010-9967-5

[52] Baykal, A., Toprak, M., Durmus, Z., Senel, M., Sozeri, H. and Demir, A. (2012) Synthesis and Characterization of Dendrimer-Encapsulated Iron and Iron-Oxide Nanoparticles. Journal of Superconductivity and Novel Magnetism, 25, 1541-1549. http://dx.doi.org/10.1007/s11051-010-9967-5

[53] Klajnart, B. and Bryszewska, M. (2002) Fluorescence Studies on PAMAM Dendrimers Interactions With Bovine Serum Albumin. Bioelectrochemistry, 55, 33-35. http://dx.doi.org/10.1016/S1567-5394(01)00170-0

[54] Lee, K. and El-Sayed, M. (2005) Dependence of the Enhanced Optical Scattering Efficiency Relative to that of Absorption for Gold metal Nanorods on Aspect Ratio, Size, End-Cap Shape, and Medium Refractive Index. Journal of Physical Chemistry B, 109, 20331-20338. http://dx.doi.org/10.1021/jp054385p

[55] Jain, P., Lee, K., El-Sayed, I. and El-Sayed, M. (2006) Calculated Absorption and Scattering Properties of Gold Nanoparticles of Different Size, Shape, and Composition: Applications in Biological Imaging and Biomedicine. Journal of Physical Chemistry B, 110, 7238-7248. http://dx.doi.org/10.1021/jp057170o

[56] Govorov, A.O. and Richardson, H. (2007) Generating Heat with Wetal Nanoparticles. Nano Today, 2, 30-38. http://dx.doi.org/10.1016/S1748-0132(07)70017-8

[57] Huang, X., Jain, P., El-Sayed, I. and El-Sayed, M. (2008) Plasmonic Photothermal Therapy (PPTT) Using Gold Nanoparticles. Lasers Medical Science, 23, 217-228. http://dx.doi.org/10.1007/s10103-007-0470-X

[58] Baffou, G., Quidant, R. and García de Abajo, F. (2010) Nanoscale Control of Optical Heating in Complex Plasmonic Systems. ACS Nano, 4, 709-716. http://dx.doi.org/10.1021/nn901144d

[59] Ghazanfari, L., and Khosroshahi, M.E. (2014) Simulation and Experimental Results of Optical and Thermal Modeling of Gold Nanoshells. Materials Science and Engineering C, 42, 185-191. http://dx.doi.org/10.1016/j.msec.2014.05.002

[60] Sassaroli, E., Li, K. and Neill, B. (2009) Numerical Investigation of Heating of a Gold Nanoparticle and the Surrounding Microenvironment by Nanosecond Laser Pulses for Nanomedicine Applications. Physics in Medicine and Biology, 54, 5541. http://dx.doi.org/10.1088/0031-9155/54/18/013

[61] Baffou, G. and Rigneault, H. (2011) Femtosecond-Pulsed Optical Heating of Gold Nanoparticles. Physical Review B, 
84, Article ID: 035415. http://dx.doi.org/10.1103/PhysRevB.84.035415

[62] Baffou, G., Quidant, R. and Girard, C. (2009) Heat Generation in Plasmonic Nanostructures: Influence of Morphology. Applied Physics Letters, 94, Article ID: 153109. 